\title{
To the theory of quasi-phase-matched parametric amplification in periodically-poled optical nonlinear crystals
}

\author{
E.V. Makeev and A.S. Chirkin \\ e-mails: makeev@newmail.ru, chirkin@squeez.phys.msu.su
}

Physics Department, M. V. Lomonosov Moscow State University, Vorob'evy Gory, Moscow 119992 Russia

\begin{abstract}
Theory of the optical parametric amplification at high-frequency pumping in crystals with a regular space modulation of the sign of nonlinear coupling coefficient of interacting waves is developed. By applying the matrix method, the theory is based on a step-by-step approach. It is shown that, in the case where the pumping intensity is less than some critical value, the spatial dynamics of the signal intensity inside a separate layer with the constant nonlinear coefficient has an oscillatory behavior and the change of the signal intensity from layer to layer is defined, in general, by the power function. The same law is valid for the change of variance of signal's quadrature components. At large number of layers, these dependences can be reduced to the well-known ones for homogeneous nonlinear optical crystals.
\end{abstract}

KEY WORDS: parametric interaction, periodically poled nonlinear crystals, quasiphase matching, step-by-step approach, quadrature component

\section{Introduction}

Quasi-phase-matched interactions of light waves as well as phase-matched interactions provide the realization of an effective energy exchange between interacting waves (see, for example, the reviews [1, 2] ) and the possibility to obtain nonclassical light [3, 4]. 
Quasi-phase-matched interactions are implemented in crystals with the periodic modulation of nonlinear susceptibility. These are crystals with the regular domain structure or periodically-poled nonlinear crystals (PPNC). In such crystals, the mismatch of wave vectors $\Delta k$ of interacting waves is compensated by an inverse-lattice vector $\kappa$ of nonlinear susceptibility's modulation

$$
\kappa=2 \pi m / \Lambda
$$

where $\Lambda$ is the modulation period and $m$ is the quasi-phase-matching order.

The conventional approach for implementing parametric interactions in PPNC consists in a choice of the domain-structure period $\Lambda$, in view of the expression

$$
\Lambda=2 \pi /|\Delta k|
$$

where $\Delta k$ is the phase mismatch between interacting waves. The period $\Lambda$ is usually determined from the analysis of the second-harmonic generation [1, 2]. At the coherence length $l_{c}=\Lambda / 2$, the second-harmonic intensity reaches the maximum value and the phase (related to the phase mismatch and equal to $\pi$ ) compensates the change of the nonlinearity sign in the neighboring layer (domain) due to the optical-axis inversion.

While nonlinear optical interactions are calculated analytically, PPNC is replaced by a homogeneous crystal with an effective nonlinear coefficient $\beta_{\text {eff }}$ distinguished from a value $\beta$ for the homogeneous crystal by the factor $2 / \pi m$, so that

$$
\beta_{\mathrm{eff}}=\frac{2}{\pi m} \beta
$$

However, such approach to the optical-parametric-amplification process is not always valued. As it is known, in a parametric process the transition to a steady state of the optimum phase relation essentially depends on the pumping intensity [5, 6. Recently, the spatial dynamics of intensity and phase of the parametrically amplified wave in PPNC was investigated in detail [7]. In particular, it was shown that at the transition from layer to layer the sign of derivative in the phase relation is changed. The change of the signal wave intensity in PPNC also differs from the homogeneous crystal case. In the present paper, the simple "step-by-step" approach to the analysis of quasi-phase-matched optical parametric amplification is presented. The knowledge of transmission factors of the 
layers is the basis of this approach. For the definition of transmission factors, the matrix approach elaborated in [8] is used. For an arbitrary number of layers with thickness equal to the coherence length, expressions for the signal intensity and variance of quadrature components for an initial random signal phase are derived.

The paper is organized as follows.

In Sec. 2 the equations for degenerate optical parametric amplification in PPNC are presented in generic form using undepleted pump approximation. Section 3 contains the solution of differential equation for the optical parametric amplification in PPNC in matrix form. In Sec. 4 the solution obtained is analyzed for the case satisfying the condition of quasi-phase-matched interactions. In Sec. 5 the possibility to generate the quadraturesqueezed light is considered when classical fluctuations are suppressed. The results obtained are summarized in Sec. 6 .

\section{Basic Equations}

The optical parametric amplification process in PPNC of a wave with frequency $\omega$ in the pumping-wave field with frequency $2 \omega$ is determined by the following equations:

$$
\left\{\begin{array}{l}
\frac{d A}{d z}=-i \beta g(z) A_{p} A^{*} \exp (-i \Delta k z), \\
\frac{d A_{p}}{d z}=-i \beta g(z) A^{2} \exp (i \Delta k z)
\end{array}\right.
$$

where $A$ and $A_{p}$ are the complex amplitudes of the signal and pumping waves, respectively, $\beta$ is the modulus of nonlinear wave coupling coefficient, $\Delta k=k-2 k_{p}$ is the phase mismatch, and $g(z)$ is the periodic function equal either to +1 or to -1 at the thickness of a separate layer, being dependent on the nonlinear-coefficient sign. In the general case, it is impossible to solve the set of equations (11) analytically.

Within the framework of undepleted pump approximation, the process under consideration is described by the equation

$$
\frac{d A}{d z}=-i \beta g(z) A_{p} A^{*} \exp (-i \Delta k z)
$$

where $A_{p}$ is the constant value. Equation (2) makes it possible to get an analytical solution on each layer for given $g(z)$. However, the solution for arbitrary number of layers, cannot 
be cast into a suitable for the application form. In this connection, to solve Eq. (2), we use the matrix method, which allows one to obtain the solution in a convenient form. Firstly we rewrite Eq. (21) introducing a reduced length $\zeta=z / L_{\mathrm{nl}}$ :

$$
\begin{aligned}
& \frac{d A}{d \zeta}=-i g(\zeta) A^{*} \exp (-i \delta \zeta), \\
& A(\zeta=0)=A_{0},
\end{aligned}
$$

where $L_{\mathrm{nl}}=1 / \beta\left|A_{p}\right|$ is the so-called nonlinear length, $\delta=\Delta k L_{\mathrm{nl}}$ is the normalized phase mismatch, and $A_{0}$ is the signal amplitude at the PPNC input.

\section{Matrix Differential Equation and Its Discrete So- lution}

Equations (3) by means of the substitution

$$
A=B \exp (-i \delta \zeta / 2)
$$

are reduced to the following system of equations:

$$
\begin{aligned}
& \frac{d B}{d \zeta}=\frac{i \delta B}{2}-i g(\zeta) B^{*} \\
& \frac{d B^{*}}{d \zeta}=-\frac{i \delta B^{*}}{2}+i g(\zeta) B
\end{aligned}
$$

which can be presented in matrix form

$$
\frac{d C}{d \zeta}=D C,
$$

with the initial values $C=C_{0}$. In Eq. (6) $C_{0}$ is the value of the matrix $C$ at $\zeta=0$

$$
C=\left(\begin{array}{c}
B \\
B^{*}
\end{array}\right), \quad C_{0}=\left(\begin{array}{c}
B_{0} \\
B_{0}^{*}
\end{array}\right),
$$

and $D$ is the matrix determined by the expression

$$
D(\zeta)=\left(\begin{array}{cc}
i \delta / 2 & -i g(\zeta) \\
i g(\zeta) & -i \delta / 2
\end{array}\right) .
$$


The function $g(\zeta)$ takes the value either +1 or -1 . One introduces matrices $D_{ \pm}$associated with these values

$$
D_{ \pm}=\frac{i}{2}\left(\begin{array}{cc}
\delta & \mp 2 \\
\pm 2 & -\delta
\end{array}\right) .
$$

Let PPNC have layers with the length $\Lambda / 2$. It is just the case used for repolarization of a nonlinear crystal. Let $g(\zeta)=1$ in the first layer. Then the solution of Eq. (6) reads

$$
C(\zeta)=\exp \left(D_{+} \zeta\right) C_{0}
$$

where the exponential function of matrix $D_{+} \zeta$ is nothing else as the transmission factor for the layer and it is introduced as follows:

$$
\exp \left(D_{+} \zeta\right)=\sum_{k=0}^{\infty} \frac{\left(D_{+} \zeta\right)^{k}}{k !}
$$

In accordance with (10), at the output of the first layer, we have

$$
C(\Lambda / 2)=\exp \left(D_{+} \Lambda / 2\right) C_{0}
$$

In order to obtain the signal at the output of the second layer, we need to suppose $D=D_{-}$ and take into account the fact that the output of the first layer is simultaneously the input of the second layer. As a result, the signal at the output of the second layer is determined by the expression

$$
C(\Lambda)=\exp \left(D_{-} \Lambda / 2\right) C(\Lambda / 2)=\exp \left(D_{-} \Lambda / 2\right) \exp \left(D_{+} \Lambda / 2\right) C_{0}
$$

For $N$ layer pairs, Eq. (13) reads as follows:

$$
C(N \Lambda)=\left[\exp \left(D_{-} \Lambda / 2\right) \exp \left(D_{+} \Lambda / 2\right)\right]^{N} C_{0}=Q^{N} C_{0}
$$

where the matrix $Q$ is

$$
Q=\exp \left(D_{-} \Lambda / 2\right) \exp \left(D_{+} \Lambda / 2\right)
$$

Thus, the matrix $Q^{N}$ defines the signal transformation from the PPNC input to its output. The solution constructed by such a way is based on the assumption that PPNC has a discrete structure. Due to this, we call such approach the step-by-step approach. 


\section{Quasi-Phase-Matched Interaction}

Now we calculate the matrix $Q$ in the case where the quasi-phase-matching condition is realized and the modulation period $\Lambda=2 l_{c}$. The exponential matrix can be presented in a compact form

$$
\exp \left(D_{ \pm} \Lambda / 2\right)=\left(\begin{array}{cc}
c+i \delta s & \mp 2 i s \\
\pm 2 i s & c-i \delta s
\end{array}\right)
$$

where functions $c(\Lambda)$ and $s(\Lambda) \operatorname{read}$

$$
c(\Lambda)=\cos (\mu \Lambda / 4), \quad s(\Lambda)=\mu^{-1} \sin (\mu \Lambda / 4),
$$

with

$$
\mu=\sqrt{\delta^{2}-4}=2 \sqrt{I_{\mathrm{cr}} / I_{p}-1} .
$$

In these relations, $I_{p}=\left|A_{p}\right|^{2}$ is the pumping intensity, $I_{\mathrm{cr}}=(\Delta k / 2 \beta)^{2}$ is the so-called critical intensity of the pumping wave or the parametric-trapping intensity. To use PPNC for the optical parametric amplification makes sense at $I_{p}<I_{\mathrm{cr}}$. In this case, the signal wave inside a separate layer oscillates. By multiplying the exponential matrices $D_{ \pm}$, the matrix $Q$ can obtained in the form

$$
Q=\left(\begin{array}{cc}
-4 s^{2}+(c+i s \delta)^{2} & 4 s^{2} \delta \\
4 s^{2} \delta & -4 s^{2}+(c-i s \delta)^{2}
\end{array}\right)
$$

Since $\Lambda=2 l_{c}=2 \pi /|\delta|$, the functions (16) are

$$
c\left(l_{c}\right)=\cos \left(\frac{\pi}{2} \frac{\mu}{\delta}\right), \quad s\left(l_{c}\right)=\mu^{-1} \sin \left(\frac{\pi}{2} \frac{\mu}{\delta}\right)
$$

At $|\delta| \gg 2\left(I_{\text {cr }} \gg I_{p}\right), \mu /|\delta| \approx 1$, therefore, we arrive at

$$
Q \approx-\frac{1}{\mu^{2}}\left(\begin{array}{cc}
-4-\delta^{2} & 4|\delta| \\
4|\delta| & -4-\delta^{2}
\end{array}\right)
$$

Raising the matrix (19) to $N$ th power, we obtain

$$
Q^{N}=\frac{1}{2}\left(\begin{array}{cc}
\alpha^{-N}+\alpha^{N} & \alpha^{-N}-\alpha^{N} \\
\alpha^{-N}-\alpha^{N} & \alpha^{-N}+\alpha^{N}
\end{array}\right), \quad \alpha=\left(\frac{|\delta|+2}{|\delta|-2}\right)
$$


Taking into account expressions (17) and (14), we arrive at the following result for $B$ :

$$
B=\frac{1}{2}\left[\left(\alpha^{-N}+\alpha^{N}\right) B_{0}+\left(\alpha^{-N}-\alpha^{N}\right) B_{0}^{*}\right] .
$$

Now in view of the relations

$$
A_{0}=a_{0} e^{i \varphi_{0}} \quad \text { and } \quad I_{0}=\left|A_{0}\right|^{2}
$$

we obtain the following expression for the signal wave intensity $I_{N}=|B|^{2}=|A|^{2}$ at the PPNC output:

$$
I_{N}=\left[\alpha^{-2 N} \cos ^{2} \varphi_{0}+\alpha^{2 N} \sin ^{2} \varphi_{0}\right] I_{0}
$$

One can see that it is just the well-known property of phase sensitivity of degenerate parametric amplification. From Eq. (22) follows that the largest amplification takes place at the signal phase $\varphi_{0}= \pm(\pi / 2)$. The largest signal suppression is achieved at the signal phase $\varphi_{0}= \pm \pi$. For number of layer pairs $N \gg 1$, at $|\delta| \gg 2$ expression (22) can be transformed into the following one:

$$
I_{h c}=\left[\cosh (\Gamma L)-\cos \left(2 \varphi_{0}\right) \sinh (\Gamma L)\right] I_{0},
$$

where $\Gamma=(4 / \pi) \beta\left|A_{p}\right|$ is the parametric amplification increment and $L=2 N l_{c}$ is the total length of PPNC. It is worthy to compare expression (23) with the analogous one for the case of a homogeneous crystal under the phase-matching condition. In the latter case, the parametric amplification increment is equal to $\Gamma_{0}=2 \beta_{0}\left|A_{p}\right|$ (see, for example, [5]), where $\beta_{0}$ is the nonlinear wave coupling coefficient for the homogeneous crystal. The ratio $\Gamma / \Gamma_{0}=(2 / \pi) \beta / \beta_{0}$ shows the difference between the optical parametric amplification in PPNC and in the homogeneous crystal. In the case $\beta=\beta_{0}$, we obtain the well-known result $\Gamma / \Gamma_{0}=2 / \pi$.

Thus, at the quasi-phase-matched-wave interaction, the intensity of parametrically amplified signal wave under the conditions mentioned above varies in the same way as in the homogeneous crystal with the effective nonlinear coefficient $\beta_{\text {eff }}=(2 / \pi) \beta$. It should be noted that the same value $\beta_{\text {eff }}$ for PPNC can be obtained, if truncated equation (2) is averaged over the modulation period of nonlinear-wave-coupling coefficient. However, in the latter case the conditions of applicability of the equations obtained are not evident. 
Using formula (22) we have calculated curves presented in Figs. 1 and 2 for $\varphi_{0}=$ $\pi / 2$. Figure 1 shows the dependence of the signal intensity on the interaction length at the quasi-phase-matched parametric amplification in PPNC. Figure 2 demonstrates the dependence of the parametric amplification increment on the interaction length at different relations between the pumping intensity and its critical value. From these figures, one can see that, at the same pumping intensity and given interaction length, the signal wave intensity increases when the pumping intensity approaches its critical value. Therefore, the replacement of PPNC by the homogeneous crystal with the effective nonlinear coefficient provides less value for the signal intensity. This difference grows up when the pumping intensity $I_{p}$ approaches to $I_{\mathrm{cr}}$. Comparison of the conversion efficiency for the parametric amplification in PPNC and in the homogeneous crystal with nonlinear coefficient $\beta_{\text {eff }}$ is done in Fig. 3. Note that at $I_{p} \simeq I_{\text {cr }}$ we have used general formula (17).

\section{Quadrature Components}

We turn now to the analysis of quadrature components $X, Y$ of the signal wave in PPNC:

$$
X(z)=\frac{1}{2}\left[A(z)+A^{*}(z)\right], \quad Y(z)=\frac{1}{2 i}\left[A(z)-A^{*}(z)\right]
$$

According to Eq. (21), one has for $X$-quadrature

$$
X(z)=\frac{1}{2}\left[\left(\alpha^{-N}+\alpha^{N}\right) \cos \phi_{-}+\left(\alpha^{-N}-\alpha^{N}\right) \cos \phi_{+}\right]
$$

where the phase $\phi_{ \pm}=\varphi_{0} \pm \delta \zeta / 2$.

To obtain a signal with suppressed fluctuations of one of the quadrature components, the random signal with a uniform distribution function for the initial phase $\varphi_{0}$ is worthy to consider

$$
w\left(\varphi_{0}\right)=\frac{1}{2 \pi}, \quad-\pi \leq \varphi_{0} \leq \pi
$$

Then the mean value $\langle X(z)\rangle=0$ and the variance of quadrature component $\left\langle X^{2}(z)\right\rangle=\overline{X^{2}(z)}-(\bar{X}(z))^{2}$ is given by the expression

$$
\left\langle X^{2}(z)\right\rangle=\frac{1}{2} a_{0}^{2}\left[\alpha^{2 N}+\left(\alpha^{-2 N}-e^{2 N}\right) \cos ^{2}(\delta \zeta / 2)\right] .
$$


At $\delta \zeta=\Delta k L=2 \pi n(n= \pm 1, \pm 2, \ldots), X$-quadrature fluctuations are reduced, namely,

$$
\left\langle X^{2}(z)\right\rangle_{\min }=\frac{1}{2} a_{0}^{2} \alpha^{-2 N}
$$

In the case $\delta \zeta=\pi(1+2 n)$, the variance reaches the maximum value:

$$
\left\langle X^{2}(z)\right\rangle_{\max }=\frac{1}{2} a_{0}^{2} \alpha^{2 N}
$$

i.e., fluctuations of quadrature components are amplified.

Thus, in PPNC the spatial changing of the variance of signal's quadrature components is determined by the power functions as well as the signal intensity. However, if number of layer pairs $N \gg 1$, expressions (27) $-(29)$ can be transformed into the forms similar to the ones for the homogeneous crystal:

$$
\left\langle X^{2}(z)\right\rangle_{\min / \max }=\left\langle X^{2}(0)\right\rangle e^{\mp \Gamma L}
$$

It is not difficult to draw a conclusion, in view of formulas (28) and (30), that suppression of fluctuations in PPNC occurs more effectively than in the homogeneous optical crystal with the effective nonlinear coefficient.

\section{Conclusions}

The application of step-by-step matrix approach allows one to calculate the optical parametric amplification in periodically-poled nonlinear crystals without analysis of the spatial dynamics inside a separate layer. The method can be applied to analysis of the processes in PPNC at an arbitrary period of the crystal repolarization and arbitrary relation of the critical intensity to the pumping intensity. In other words, the method is not restricted to the conditions $I_{p} \ll I_{\mathrm{cr}}$ and $N \gg 1$ which are necessary for the correct analysis in the traditional-approach case where PPNC is replaced by an homogeneous crystal with

effective nonlinear coefficient. This circumstance allows one to use this method for both analytical and numerical [8] calculations.

The spatial dynamics of the signal intensity and quadrature components was analyzed versus the ratio $I_{p} / I_{\mathrm{cr}}$ and the interaction length. It was shown that at the pumping 
intensity less than the critical value, the spatial distribution of the signal intensity inside a separate layer exhibits an oscillatory character and changing of the signal intensity and variance of quadrature components from layer to layer follow the power function dependences. In the case of larger number of layers and $I_{p} \ll I_{\mathrm{cr}}$, these dependences can be reduced to well-known formulas for homogeneous nonlinear crystals. Notice that the requirement $I_{p} \geq I_{c r}$ corresponds to the parametric trapping in the homogeneous crystal.

It should be emphasized that our analyze has shown that the quasi-phase-matched optical parametric process in PPNC occurs, generally speaking, more effectively than the parametric process in the homogeneous crystal with the nonlinear coupling coefficient equal to the effective nonlinear coefficient for PPNC. The generalization of the approach presented here onto the case of quasi-phase-matched consecutive wave interactions and nonstationary parametric interactions is in progress.

\section{Acknowledgments}

The authors are grateful to I. V. Golovnin, G. D. Laptev, E. Y. Morozov, and A. A. Novikov for helpful discussions.

The work was partially supported by INTAS under Project No. 01-2097.

\section{References}

[1] R. L. Byer, J. Nonlin. Opt. Phys. Mater., 6, 549 (1997).

[2] A. S. Chirkin, V. V. Volkov, G. D. Laptev, and E. Yu. Morozov, Quantum. Electron., 30, 847 (2000).

[3] P. Baldi, P. Aschieri, S. Nouh, M. De Micheli, D. B. Ostrowsky, D. Delacourt, and M. Papuchon, IEEE J. Quantum Electron., 31, 997 (1995).

[4] O. K. Serkland, M. M. Fejer, R. L. Byer, and Y. Yamamoto, Opt. Lett., 20, 1649 (1995). 
[5] S. A. Akhmanov, Yu. E. Diakov, and A. S. Chirkin, Introduction In Statistical Radiophysics and Optics [in Russian], Nauka, Moscow (1981).

[6] A. S. Chirkin and D. B. Yusupov, Quantum. Electron., 8, 440 (1981).

[7] E. V. Makeev and A.S. Chirkin, Opt. Spectroskop. (in press).

[8] V. Beskrovnyy and P. Baldi, Opt. Exper., 10, 990 (2002). 


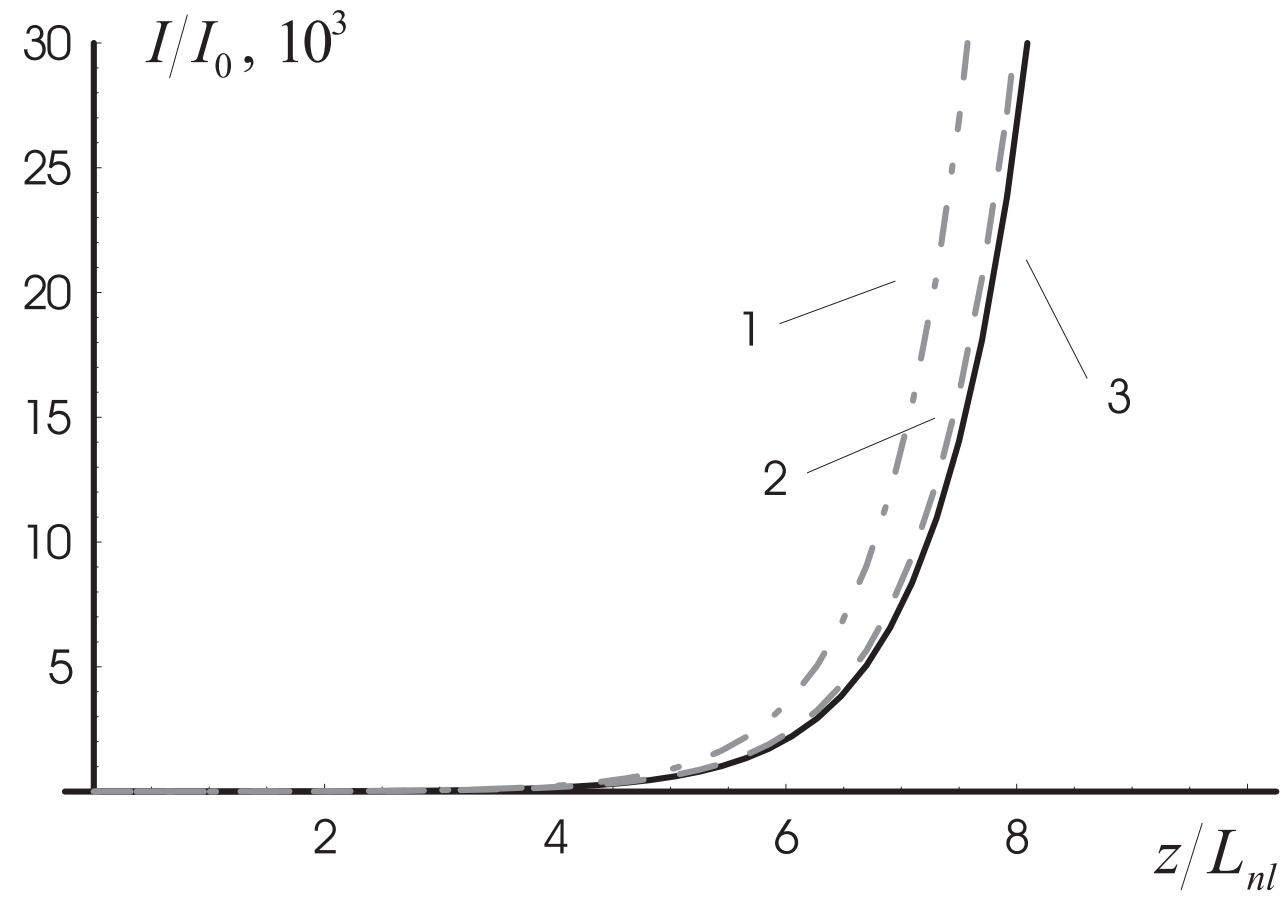

Figure 1: Coefficient of the parametric signal amplification in PPNC as a function of the interaction length for the phase $\varphi_{0}=\pi / 2$ and different ratios of the pumping intensity $I_{p}$ to the critical intensity $I_{\mathrm{cr}}: I_{p}=I_{\mathrm{cr}}(1), I_{p}<I_{\mathrm{cr}}(2)$, and $I_{p} \ll I_{\mathrm{cr}}(3)$. Curve 2 corresponds to formula (22) and curve 3 corresponds to formula (23). 


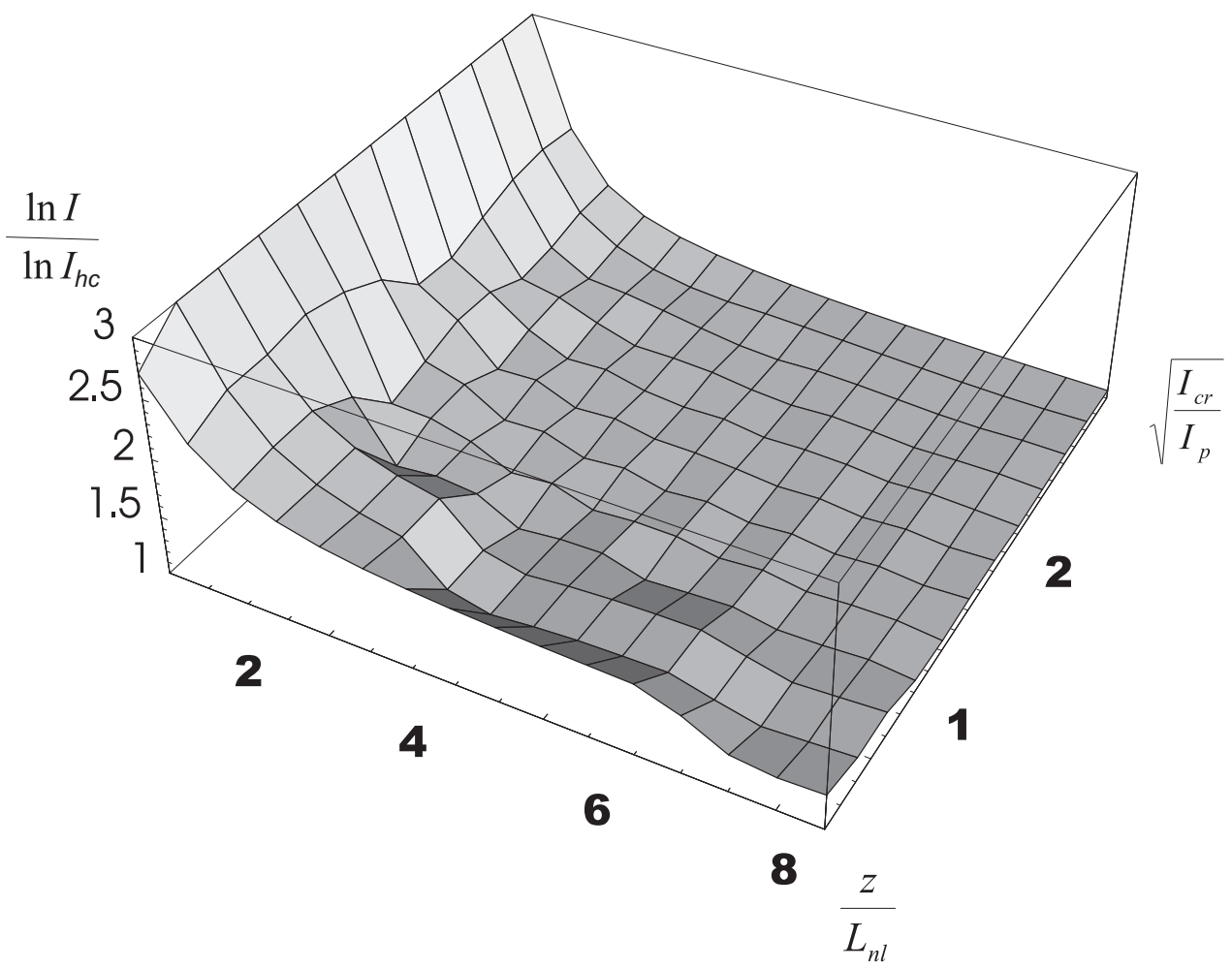

Figure 2: Relative increment of the parametric amplification in PPNC as a function of the interaction length and ratio $I_{\mathrm{cr}} / I_{p} . I$ and $I_{\mathrm{hc}}$ are the signal intensities for the case of PPNC and homogeneous nonlinear crystal, respectively. 


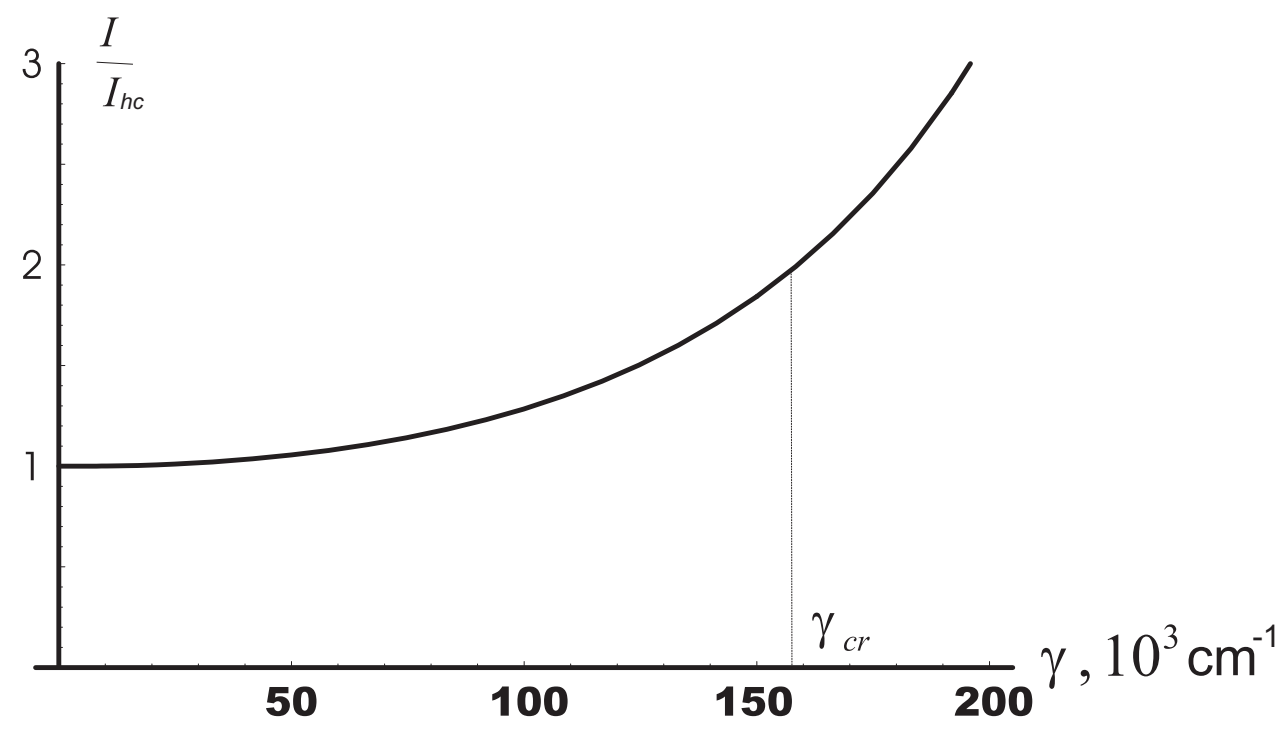

Figure 3: Ratio of the parametrically-amplified signal intensity $I$ in a $\mathrm{LiNbO}_{3} \mathrm{PPNC}$ to the intensity $I_{\mathrm{hc}}$ in a $\mathrm{LiNbO}_{3}$ homogeneous crystal with corresponding effective nonlinear coefficient $\beta_{\text {eff }}$ versus $\gamma=\beta \sqrt{I_{p}}$ for the eee-interaction type and $\lambda=0.5 \mu \mathrm{m}, \Lambda=20 \mu \mathrm{m}, N=3$, and $\gamma_{\mathrm{cr}}=\beta \sqrt{I_{\mathrm{cr}}}$. 